\title{
Evaluation of Development of Sharing Economy from the Perspective of Social Network
}

\author{
Yichen Yang \\ School of Politics and Public Administration, South China Normal University, Guangzhou 510006, Guangdong, China \\ Email: 2658913783@qq.com
}

\begin{abstract}
This article focuses on the study of the macro and interdisciplinary aspects of the sharing economy. Based on the perspective of social networks, the overall evaluation of the development of this new economic form is carried out, with a view to exploring the sound development of the sharing economy with a new perspective of sociology. First of all, the sharing economy has changed the structure and relationships of social networks. However, social networks have negative effects on trust mechanism, heterogeneous connection, social governance, individual autonomy and capital circulation in the considerable changes. Based on the concern for these negative effects, this article still launches the development strategy of the sharing economy in the aspects of traditional relations, institutional supply, lowering barriers, abandoning inertia, and identifying social capital from the perspective of social network.
\end{abstract}

Keywords: social network, sharing economy, social capital

\section{Introduction}

The inspiration of this article comes from the article "Burger is a miracle", which explains that in today's social network pattern, individuals are no longer completely independent, but we are interdependent and we share collective knowledge and skills. This sharing makes me to think of a hot word that is very related to it - sharing economy. Sharing economy, while re-coordinating market production, distribution, circulation and consumption, is also reshaping "the network connection relationship from competition to cooperation, from power to circle, from organization to community". Producers, consumers and traders are linked by the network, and the social ecosystem formed by this provides a research direction for how to optimize the allocation of social resources in social relations.

Through searching and reading a large number of previous literature on sharing economy, the author found that the current research on sharing economy at the micro level and in the economic field is more common, which is based on the development of innovative enterprises, the analysis of a specific sharing platform, in-depth development of a shared resource case and so on. But in the macroscopic aspect and the interdisciplinary field research is relatively deficient. As a new economic model, sharing economy has been more favored by economic researchers. In fact, in addition to the important influence on the economic life, sharing economy from the perspective of sociology, sharing economy, in a unique organization, provides the social interaction between different groups with more channels and opportunities, its distinctive social network structure will also be based on interpersonal relationship, the trust system, many aspects of influence social capital flows, etc. However, there are few papers on the topic of social network and sharing economy, and they mainly study the following aspects.

(1) This paper makes a detailed data analysis on the structure, theme and trend of the sharing economy research under the social network, so as to find out the blind spots and shortcomings of the current research on the generic economy, and broaden the content available for research on the sharing economy in the future through the use of special software.

(2) Deeply explore the internal hierarchical, hierarchical and hierarchical nature of the social network of the sharing economy; deconstruct the connection and decomposition modes in the social network of the sharing economy. Then explore the value connotation and value transfer process of the sharing economy business model based on the network structure; construct the sharing economy value network analysis framework based on the structural hole theory; conduct exploratory theoretical research on how the sharing economy realizes value creation through the network structure.

(3) From the perspectives of interpersonal relationships, employment and belonging relationships between individuals and groups, and the mobility of different classes of groups, the importance of the sharing economy development is analyzed in alleviating social labeling, enhancing social trust, forming diverse employment relationships, resetting social identities and belonging, and smoothing flow channels, etc. Thus, the conclusion is drawn that "sharing economy reconstructs social relations". 
(4) Through drawing the social network structure diagram, we can get the changes of social network structure and relationship under the Internet link. In addition, the negative effects of social network under the sharing economy are briefly discussed from the perspectives of network role and network dependence.

Some of the above literature failed to clearly reveal the relationship between the sharing economy and social network; some were still influenced by the traditional mass studies and put a large impact and strategic analysis on the sharing economy; some interpreted the sharing economy by using social network as an analytical tool. On the other hand, based on the exploration of the relationship between sharing economy and social network, the author puts the development of sharing economy in a new perspective by reviewing and looking forward to the research on social network theories, including Granovetter's weak relationship theory, Bian Yanjie's strong relationship theory, Burt's structural hole theory and social capital theory. The social network perspective.

\section{Changes of social network under the sharing economy}

\subsection{Changes in social network structure}

\subsubsection{Network structure attributes: from centralized network to incomplete distributed network}

The traditional social network structure is a network structure in which resources and information are controlled by a central system and flow to individuals individually through each node. In the structure of centralized social network, there is no communication bridge between nodes, and individuals far away from the center are less connected. The emergence of sharing economy has broken the pattern of single resource control and replaced it with distributed social network.In this structure, there will be multiple centers and multiple nodes, which is conducive to realize the two-way flow of information resources under the connection of multiple nodes, and constantly expand the social network. But the word of "incomplete", because of the shared platform both via the Internet more than the advantages of manufacturing center and node, but at the same time, due to its own economic attributes, also make it in expanding social network at the same time, by actively promote their own brands, services, user experience, popularity and so on, to absorb more individuals involved in the building of social networks. In this case, high-quality social capital will accumulate, and social networks also show a trend of "centralization".

\subsubsection{Network structure features: enhanced heterogeneity and enhanced overall centrality}

Sharing economy is committed to build the whole network structure. The link of social network is enhanced, which makes the individuals who cannot form the link establish the link. At the same time, the role of the original part as a bridge is weakened, which makes up for the structural hole in the low-level social network. The center distribution of social network is more balanced, and the overall centrality will be improved. Moreover, as people of different classes, occupations, and ages are connected, the heterogeneity of social networks increases.

\subsection{Changes in social network relationships}

\subsubsection{Weak ties can be strengthened and expanded}

Sharing economy has shaped a new social structure — network community, which is a process of reconstructing a strong relationship on the basis of a weak relationship. Two individuals or groups that do not know each other can use the Internet platform to achieve social interaction - to share or reuse unused and underused assets, knowledge, skills, information and other resources. In the process of increasing social interaction, weak ties are strengthened, and individual social networks continue to extend outward with various transaction behaviors.

\subsubsection{Part of the asymmetric "feeling based" transaction is transferred to the market transaction with both rights} and responsibilities

The social relationship based on the geographical location, blood relationship and industry relationship in traditional Chinese society is essentially a "human" relationship, and the trading relationship derived from it is also asymmetric. That is, the trading behavior often focuses on a reciprocity of sharing behavior, and the contract formed between the two parties is full of "human".

This kind of asymmetric "favor" transaction has several disadvantages. (1) The supply of social capital is simple, and it is difficult to accurately meet the needs of the other party. (2) The contract dominated by "human favor" lacks the constraint of legal system, and the interests of both parties in the transaction cannot be effectively guaranteed. (3) Unjustified reciprocity and mutual benefit is the product of excessive human handling, and may even breed corruption, infringement and other social problems. By building several sharing platforms and relying on the technological advantages of the Internet, sharing economy makes individual social networks more extensive and dense, information transmission and flow more convenient, resource supply and demand more personalized and diversified, and there is more space for individuals or groups 
to choose. Moreover, the intervention of the legal system makes the interaction between the two parties subject to necessary supervision and protection. In contrast, part of the asymmetric "favor" transaction will be replaced by the market exchange with the coexistence of rights and obligations.

\subsubsection{The continuous extension of social trust network}

The trust network is not only based on the trust between strong relationships, but also based on the trust between strangers, which makes the social trust network greatly extended.

As one of the representatives of the sharing economy, Airbnb not only provides a platform for voluntary transactions between the supply side and the demand side, but more importantly, it explores the goodness of human nature to build a trust relationship between strangers, so that hosts and guests can create a new form of life under the dual action of emotion and system. The interaction between the landlord and the tenant of SunnyHome is just like this. The landlord Sunny provides a room with a story for the tenant, and the tenant shares the meaning and value of the story with the feedback from the landlord. The interaction between them has reached the level of spiritual resonance. The guest wrote: "I am also amazed by Sunny's experience and seeing. I have been to so many places that I have been longing for for a long time and have never even heard of. I think this is what I want to pursue in the future." What they have created together on Airbnb's platform, based on a sense of identity with each other's way of life, is a trust that goes beyond transactional relationships.

\section{Negative effects of social networks in the sharing economy}

\subsection{Trust mechanism is damaged due to avoidance of "bad reviews"}

The establishment of trust mechanism under the sharing economy depends on the continuous information feedback of consumers. The relative distribution of "good" and "bad" reviews will affect the judgment of the demand side on the level of trust of the supply side, thus affecting the transaction decisions of the demand side and the economic interests of the supply side. Every supplier wants "good" reviews everywhere, and "bad" reviews nowhere. It is precisely this instinctive expectation that makes some suppliers choose to delete "bad reviews" or hire water army to "inject water" to praise in order to eliminate the negative influence of "bad reviews" to themselves. When users make the choice of transaction parties, affected by the precedent of dishonest behavior of some suppliers, users will hold a kind of uncertain and fuzzy mentality towards the "favorable" information presented, which will further aggravate the trust crisis and destroy the trust mechanism in the social network.

\subsection{The filling process of structural holes can easily lead to redundant connections}

The social network under the sharing economy has been developing dynamically in the process of constantly filling the structural holes and striving to form the ideal fully connected network, that is, the network that connects the whole individuals in the world. According to Burt's theory, in a network without structural holes, knowledge, information and other resources among members are highly repetitive and redundant due to the direct connection between network members. This kind of social network with high homogeneity is not conducive to the appreciation of individual social capital in terms of quality and quantity. Therefore, in the social network, a large number of homogenous connections are redundant connections.

\subsection{The increasing complexity of social governance}

In the open and innovative sharing network environment, social individuals or groups can rely on their existing assets, knowledge, skills, information and other resources to realize the multiple exchange of social roles through the leaping of different fields and the docking of different needs, so that social capital can flow rapidly among all social classes. However, precisely because of the complexity of subject identity and the universality of social capital flow, it also intensifies the complexity of social governance.

\subsection{Individuals and groups are more dependent on social networks}

Due to the increasingly expanding social network individuals, groups, such as assets, knowledge, skills, information can be fully shared state, interdependence, mutual dependence between individuals and groups of contact will be strengthened. The "everything" sufficient supply can meet the needs of the individuals and groups on social capital. But at the same time, the dependence of individuals and groups on social networks also increases. When facing problems that need to be solved in daily life, perhaps the convenience of sharing will be transformed into a kind of inertia in people's thinking, which drives people to first choose "turning to others" rather than "self-reliance", thus weakening the autonomy of individuals and groups.

When this dependence reaches a certain intensity, it may enable us to ignore the consideration of our own situation, thus affecting our decision-making judgment. As a result, this kind of dependence mainly comes from our demand side's search for problem-solving ideas. In short, we are eager to directly see the method guidance, so this kind of increasing dependence 
mostly corresponds to the social capital in terms of knowledge and skills, rather than the social capital in kind.

\section{5 "Sharing barriers" still exist, and complete sharing is difficult to achieve}

Whether explicit barriers or hidden barriers, there are obstacles to the free circulation of social capital. Dominant barriers mainly embodies in for the customer to look for, suppliers will be in black and white to identify requirements, these requirements may be individuals or groups to education, work experience and achievement of the requirements. For those at the stage of growth, there is no accumulation of the people. They are hard to participate in the sharing of high levels of social capital. And hidden barriers are above the internalization of "black and white" requirements, the suppliers may be at heart has set up a set of standard and preference. When asked, the user will beat about the bush to some basic information about the user's first, and then contrast the standard and preference, so as to decide whether to share.

In addition to the fact that there may be explicit or implicit barriers to sharing between the supply side and the demand side. Because shared platforms are in a dominant position in social networks — bridging contact points occupying the position of structural holes in social networks in the era of big data. The derived social capital includes organizational ability, market network and customer relationship, etc., which creates new information asymmetry with the supply and demand sides connected at both ends. Such information asymmetry makes both the supply and demand parties under the potential control of the platform, and it is difficult for them to fully participate in the shared social interaction.

\section{Development countermeasures of sharing economy from the perspective of social network}

\subsection{Pay attention to traditional relations under the sharing economy}

Although part of the assignment of the "human" trading asymmetry in power and responsibility and save the market transactions, but this does not mean that the traditional pattern of difference sequence under the shared economy lost the value of existence. On the contrary, the pattern of difference in the social network can make the individual has a chance to act as nodes, become channels of transmission of information to more people, bring new value for sharing economy.

This can be understood in the context of our personal experience. For example, when a user on a platform with a good trading experience, the people beside also have the same demand (may be eager to lease some items), then based on the traditional "practice, kiss, letter" relationship, the user will be very consciously and actively take the last time the experience sharing and recommend to others, in an attempt to revenue sharing with others. When the recommended person tries it out, he or she may have a good trading experience and then share it with others in his "circle" who need it. In fact, the value of the traditional pattern of differential order in the sharing economy is worthy of attention and in-depth exploration. Although it does not directly expand the social network of individuals, it shares the benefits of the sharing economy with others (of course, those who are "familiar, close and trustworthy") to some extent.

\subsection{Matching the institutional supply of the sharing economy}

Matching the system supply of the sharing economy involves a lot of content. Based on some risks in the sharing economy, from the perspective of macro-control, the sharing economy needs to solve the problems of market monopoly and unfair competition, and the information security of social members.From the perspective of standardizing the market subject, it is necessary to determine the market subject status and relevant rights and obligations of the platform. From the perspective of labor relations, it is necessary to pay attention to the new mode of guaranteeing the rights of labor subjects in the sharing economy under the multi-network structure.Based on the negative effect of social network in the sharing economy, the system supply optimization and integration of the above aspects are as follows.

\subsubsection{The shared platform shall form an independent all-round evaluation mechanism}

Since the supplier has an instinctive expectation for "favorable comments", the sharing platform should form an independent all-round evaluation mechanism in order to prevent such deviant behaviors as deleting "bad comments" and injecting "favorable comments" that damage the trust mechanism under the profit-seeking mentality out of control. "Independent" is because of the unique advantage shared platforms have in social networks — the bridging points that occupy the structural holes. As a result, shared platforms can view the whole transaction behavior from the perspective of "God" and know the strengths and weaknesses of both suppliers and suppliers. It is precisely this asymmetry of information between the platform and the supply and demand parties that can be a great opportunity for the shared platform to rationally evaluate the entire transaction behavior. This can moderately reduce the negative impact of misleading evaluations displayed by the supply side, and replace them with more comprehensive, rational, and holistic evaluations, and reconstruct the trust mechanism of social networks in the sharing economy. 


\subsubsection{The government should form a rigorous information security guarantee mechanism}

Due to the precise matching between supply and demand and the trust of both parties, both parties will disclose part of their personal information to each other and the platform. Under the existing illegal information trading market, the government should strengthen institutional norms and binding supervision on relevant key industries, especially insurance marketing, real estate agency, telecom operation, logistics and express delivery, investment information consultation and other industries, to prevent the leakage and abuse of user information at the source.

In addition, the government will step up efforts to crack down on the disclosure and abuse of users' personal information and other acts that harm consumers' rights and interests, while strengthening the protection, creation, application and service of intellectual property rights related to the development of the sharing economy, including patents, copyrights and trademarks.

The rigorous information security guarantee mechanism depends not only on the system construction, but also on the advantages of science and technology. Committed to fully implement the data security and usability of day letter data leakage protection system (TopDLP), in-depth content recognition technology as the core, the network level, terminal level of multidimensional ways and content monitoring and identification and control, to the file server, database, storage server for sensitive data storage detection and protection. Locate and protect the storage locations of important sensitive data, ensure the compliant use of sensitive data, prevent active or accidental data leakage, and comprehensively protect user information from terminal, network and storage.

4.2.3 The government should form a governance mechanism on the basis of clarifying the governance object and governance subject

In view of the aggravation of the complexity of social governance under the sharing economy, the government should first clarify the governance object and governance subject. Because we are in a highly interconnected world under the shared economy, social capital will continue to happen quantitative change and qualitative change, groups and individuals in the social network will has the quantitative change and qualitative change unceasingly. Therefore, the management object is not a determine the object of the same, on the contrary, it should be a dynamic open network.

Secondly, when we regard the object of public governance as an open network, it is natural that the subject of governance is no longer a single subject, but includes any actor that affects the network structure. Here, these actors may themselves be the nodes that constitute the network structure of governance objects, or they may be the new nodes triggered by the evolution of governance objects.

In the clear governance object, on the basis of the governance body, the government needs to identify the shared economy and social network structure influence of different nodes, and then, the government needs to analyze the mechanism of action of different nodes on the network properties, prompting more influential nodes network hub position, and through the node interaction to promote optimization and upgrading of the whole social network, forming a governance mechanism that pays attention to network nodes and their interactive processes.

\subsection{Lowering "sharing barriers" and breaking down "hidden barriers"}

As far as possible, the supplier should not focus solely on the achievements of individuals or groups such as educational background and work experience, but should give consideration to the sincerity and potential of the demand side in the interaction and communication, and give those who are still in the growth stage and have little accumulation an opportunity to participate in the sharing of high-level social capital.

For the requirements of the customers that need to be sought, the supplier should best present them in "black and white" explicit way, so that the disclosure can not only make the customer clear at a glance, but also can avoid the arbitrary change of the subjective standard in the heart of the supplier, which is conducive to breaking down the "hidden barrier" and increasing the opportunity of trading.

\subsection{Individuals should make good use of sharing convenience and discard "thinking inertia"}

When they encounter problems, they should first analyze them based on their own situation, record the methods or doubts that they have not thought of yet, and then tap into the social network to mine relevant social capital as assistance, so as to form a solution in line with their own situation while abandoning them.

\subsection{Individuals and platforms should improve their ability to identify social capital}

An ideal network without structural holes brings opportunities for social members to contact directly, resulting in highly repetitive and redundant resources such as knowledge and information among members. In order to improve the heterogeneity of social networks and reduce redundant connections, it is necessary to increase the value of social capital in both quality and quantity. First of all, sharing platform after to classify the social capital, shall be of the same kind of 
social capital for further identification, completion of segmentation, information platform can moderate let suppliers add some comments, or in identifying the suppliers after the innovation of the social capital value, the practical value of actively expand the propaganda, guide the demand to deal. As the demander, individuals or groups should rationally examine the social capital presented, improve their ability to identify the innovative value and practical value of the same kind of social capital, and minimize the interference from the same kind of resources.

\section{Conclusion}

\subsection{Social networks have changed in structure and relationship under the sharing economy}

In terms of structure, the heterogeneity and centrality of incomplete distributed social networks are enhanced. In terms of relationships, market transactions with both rights and responsibilities are more favored, and weak ties are strengthened and expanded, so that trust can be conveyed between strangers.

\subsection{Negative effects of social networks under the sharing economy}

Due to the instinct of pursuing profit, the complexity of subject identity and the universality of social capital flow, the social network under the sharing economy has negative effects on trust mechanism, heterogeneous connection, social governance, individual autonomy and capital flow.

\subsection{Discussion on the development path of the future sharing economy}

For more active revenue sharing, the traditional relationship under the pattern of differential order is still worthy of attention. The emergence of negative effects warns the government and platforms to match the institutional supply from evaluation, information security guarantee to social governance. The supply side and the demand side represented by individuals and groups in the transaction should also make some contribution. The supply side should reduce the single achievement evaluation and appropriately give more people the opportunity to participate in the sharing. At the same time, explicit requirements are conducive to breaking down "hidden barriers". On the other hand, individuals should not only abandon the thinking inertia of "asking for help", but also improve their ability to identify social capital with the help of the integration of platform priority.

\subsection{Lack of research}

This article only focuses on a more macroscopic and theoretical perspective, and through literature reading and case collection, it sorts out the innovations, possible problems and strategies in the development of the sharing economy in more detail. However, due to the theoretical foundation and limited space, for some specific emergencies, or different types of shared enterprises (supply side), individuals of different occupations (demand side) should be explored and screened in the social network of the sharing economy. The use of social capital, including in-depth analysis of specific cases, etc., is not covered in this article, and further research and discussion are still needed in the future.

\section{References}

[1] Chen Zhangwang, Yang Yuping. The research structure and trend of sharing economy based on social network[J]. Journal of Nanjing University of Aeronautics and Astronautics (Social Science Edition). 2020; 22(01): 45-52.

[2] Yang Ge. Information Linking, Technology Embedding and Network Effect: Social Networks in Sharing Economy[J]. Zhejiang Social Sciences. 2019; (12): 66-72+93+157.

[3] Gao Suying, Zhang Ye, Liu Hongbo. The social network analysis of business model value creation of sharing economy[J]. Journal of Technical Economics \& Management. 2019; (07): 79-84.

[4] Cui Yu. (2019) Policy Choice of Shared Economy Governance[D]. East China University of Political Science and Law, Shanghai.

[5] Cheng Xiaole. (2019) Research on structure optimization of shared healthcare stakeholder network[D]. Chengdu University of Technology, Chengdu.

[6] Cao Erbao, Yu Man, Bi Gongbing. Socialized operation management: An emerging research field[J]. Journal of Management Sciences in China. 2018; 21(11): 112-126.

[7] Tang Limin. (2018) Research on business model innovation mechanism of enterprises in sharing economy[D]. Capital University of Economics and Business, Beijing.

[8] Zhang Chi. (2018) A Game analysis of social networks and shared platforms[D]. Southeast University, Nanjing.

[9] Zhao Shuliang. (2016) Research on regional open innovation and resource sharing mode in the context of internet[D]. University of Science and Technology of China, Hefei. 
[10] Zhang Xiu'e, Zhang Haoxuan. Review and prospect of social network theory[J]. Modern Business. 2018; (20): 154-157.

[11] Cai Dandan, Yu Fengxia. Sharing economy restructures social relations[J]. E-government. 2016; (11): 12-18. 